\title{
Development and validation of a predictive nomogram for postoperative osteonecrosis of the femoral head with cannulated screws fixation
}

\author{
Wanbo Zhu ${ }^{1,2}$, Kai Xie ${ }^{2}$, Xianzuo Zhang ${ }^{2} \wedge$, Jiazhao Yang ${ }^{2}$, Lei Xu' ${ }^{2}$, Junchen Zhu ${ }^{3}$, Shiyuan Fang ${ }^{1,2}$, \\ Chen $\mathrm{Zhu}^{2}$
}

${ }^{1}$ Department of Orthopaedics, Affiliated Anhui Provincial Hospital of Anhui Medical University, Hefei, China; ${ }^{2}$ Department of Orthopaedics, the First Affiliated Hospital of University of Science and Technology of China, Hefei, China; ${ }^{3}$ Department of Orthopaedics, the Second Affiliated Hospital of Anhui University of Chinese Medicine, Hefei, China

Contributions: (I) Conception and design: X Zhang; (II) Administrative support: S Fang, C Zhu; (III) Provision of study materials or patients: J Yang, L Xu; (IV) Collection and assembly of data: W Zhu, K Xie; (V) Data analysis and interpretation: W Zhu, J Zhu; (VI) Manuscript writing: All authors; (VII) Final approval of manuscript: All authors.

Correspondence to: Xianzuo Zhang; Shiyuan Fang; Chen Zhu. Department of Orthopaedics, the First Affiliated Hospital of University of Science and Technology of China, Hefei, China. Email: zhangxianzuo@foxmail.com; fangshiyuan2008@126.com; zhuchena@ustc.edu.cn.

Background: Osteonecrosis of the femoral head (ONFH) remains a major complication of femoral neck fractures. Early interventions require preliminary prediction and detection. In this study, we aimed to evaluate the perioperative variables of postoperative ONFH in femoral neck fracture patients with closed reduction and cannulated screw fixation. We also established and validated an individualized nomogram for the prediction of postoperative ONFH.

Methods: We included 470 patients with ONFH from two hospitals [First Affiliated Hospital of University of Science and Technology of China $(n=360)$ and Southern Branch of the First Affiliated Hospital of the University of Science and Technology of China $(n=110)]$. We evaluated the prognostic value of multiple perioperative variables using a Cox regression model in the training cohort. We developed a nomogram for the prediction of $\mathrm{ONFH}$ using a logistic regression model. We assessed the performance of this nomogram in a validation cohort and evaluated its clinical value.

Results: Of the 470 patients who met the inclusion criteria, 141 (30.0\%) developed postoperative ONFH. We found alcohol use [odds ratio (OR), 1.743, 95\% confidence interval (CI), 1.042-2.901, P=0.033], cerebrovascular disease (OR, 5.357, 95\% CI, 2.318-13.13, $\mathrm{P}<0.001)$, interval to surgery (OR, 5.273, 95\% CI, 2.724-10.43, $\mathrm{P}<0.001$ ), Garden classification (OR, 23.17, 95\% CI, 6.812-145.3, $\mathrm{P}<0.001$ ), Garden index (OR, 5.935, 95\% CI, 2.670-14.184, $\mathrm{P}<0.001$ ), interval to partial weight-bearing (OR, 0.053, 95\% CI, 0.006-0.296, $\mathrm{P}=0.002$ ), and six-month Harris hip score (OR, 0.856; 95\% CI, 0.792-0.919, $\mathrm{P}<0.001$ ) were independent predictors of postoperative development of ONFH. Based on these variables, we developed a nomogram that showed good discrimination in both the training [area under the curve (AUC) $=0.865$ ] and the validation cohort (AUC =0.877). The favorable performance of this nomogram was also confirmed in the validation cohort.

Conclusions: We developed and validated an easy-to-use nomogram for predicting postoperative ONFH. This nomogram can aid decision-making of intraoperative interventions and postoperative rehabilitation plans for patients, surgeons, and osteo-rehabilitative physicians.

Keywords: Osteonecrosis of the femoral head (ONFH); femoral neck fracture; prediction model; nomogram

^ ORCID: 0000-0002-6432-7745. 
Submitted Jun 21, 2020. Accepted for publication Nov 27, 2020.

doi: $10.21037 /$ atm-20-4866

View this article at: http://dx.doi.org/10.21037/atm-20-4866

\section{Introduction}

Femoral neck fractures are very common and constitute more than half of all hip fractures (1). It has been estimated that there are more than 1.6 million cases of femoral neck fractures worldwide each year, which continue to rise due to aging societies and motorization $(2,3)$. Femoral neck fractures are associated with a mortality of over $20 \%$ and a large number of disabilities. The optimal surgical treatment for femoral neck fractures remains unclear (4). Although revision surgery rates of approximately $35 \%$ have been reported after internal fixation, it is still the preferred choice for patients under 65 years of age and for most non-displaced fractures $(5,6)$. One of the most common complications of femoral neck fractures after internal fixation is osteonecrosis of the femoral head (ONFH), occurring in $20-37.9 \%$ of patients (7-9). Postoperative ONFH results in hip disability, pain, and severely reduced quality of life (10). Preliminary ONFH can be delayed or reduced by a series of interventions $(11,12)$. However, this requires early diagnosis and prediction of postoperative ONFH in individualized patients.

A large number of studies have been performed using preoperative patient risk factors to predict $\mathrm{ONFH}$ after internal fixation (13-15). Although satisfactory results were obtained in these studies, intervention strategies could not be individualized to avoid or reduce the development of postoperative ONFH. Additionally, the adjusted interventions during and after surgery could not be incorporated into the prediction system to improve the final predicted incidence. Thus, there is a need for a new prediction model based on perioperative variables to aid surgeons and orthopedic rehabilitation physicians in decision-making. This is a retrospective study where we evaluated multiple perioperative risk factors associated with postoperative ONFH after internal fixation. We also developed a nomogram that predicts the individual risk of ONFH in patients with internal fixation, providing a decision basis for surgeons, rehabilitation physicians, and patients. We present the following article in accordance with the TRIPOD reporting checklist (available at http:// dx.doi.org/10.21037/atm-20-4866).

\section{Methods}

\section{Patient population}

In this study, we retrospectively collected data from 671 patients who sustained a femoral neck fracture treated by closed reduction and cannulated screw fixation between January 2009 and June 2017. Patient data were collected from two urban tertiary hospitals: the First Affiliated Hospital of University of Science and Technology of China (FAH, n=481) and the Southern Branch of the First Affiliated Hospital of the University of Science and Technology of China (SBH, $\mathrm{n}=190)$. The inclusion criteria were as follows: (I) patients aged over 18 years with a fresh femoral neck fracture; (II) continuous follow-up for at least three years and availability of clinical characteristics. The exclusion criteria were as follows: (I) pathological fracture or bilateral fractures; (II) long-term hormone use; and (III) removal of internal implants in other hospitals. Postoperative ONFH is diagnosed by pelvic MRI or codiagnosed by three experienced orthopedic surgeons based on the last follow-up pelvic radiograph. Of the 671 patients, 201 were excluded: 144 were lost to follow-up, 31 did not undergo a pelvic MRI or radiograph in their last follow-up, 14 underwent implant removal surgery in other hospitals and there was surgery information lacking, 10 received long-time hormone treatment, and 10 were diagnosed with a pathological fracture. Ultimately, 470 patients were included in this study and classified as with or without ONFH. The study was conducted in accordance with the Declaration of Helsinki (as revised in 2013). This study was approved by the Ethics Committee of the First Affiliated Hospital of University of Science and Technology, the common ethics review institution of the First Affiliated Hospital of University of Science and Technology and the Southern Branch of the First Affiliated Hospital of the University of Science and Technology of China (No. 2020-P-049). The individual consent for this retrospective analysis was waived.

Baseline clinical data were derived from medical and follow-up records (16) and included age (17), sex, body mass index (BMI), injury site, tobacco use, alcohol use (18), cause of injury, preoperative blood tests (19-21), classification of 
risk of anesthesia according to the American Association of Anesthesiologists (ASA) classification, Charlson's weighted index of comorbidities (WIC), injury severity score (ISS) score, cerebrovascular disease, interval to surgery, preoperative Garden classification, Pauwels angle (22), postoperative Garden index, postoperative interval to partial weight-bearing, postoperative interval to full weightbearing, and six-month postoperative Harris hip score.

\section{Hospitalization management and rehabilitation}

All patients were hospitalized in either general or day care wards for surgery. Preoperative evaluation and blood tests were performed after admission. Fracture patterns and severity, including Garden classification and Pauwels angle, were evaluated based on preoperative images of pelvic radiography and computed tomography $(\mathrm{CT})$. All patients wore an anti-rotation shoe on the injured limb before surgery. Preoperative traction was not routinely carried out in patients in either hospital.

Surgeries were carried out as soon as possible after hospitalization. All surgeries were performed under general anesthesia and operated by a major surgeon team using closed reduction with three cannulated compression screws fixed in an inverted triangle configuration, following guidelines from the American Academy of Orthopedic Surgeons (23). Intraoperative traction tables were routinely used. Patients who underwent open reduction due to poor closed reductive quality were excluded from this study. Postoperative radiography was performed. Anticoagulant therapy and rehabilitation were guided by orthopedic rehabilitative clinicians. Continuous passive motion on the bed was permitted on postoperative day 1 , and weight-bearing was not encouraged for one month in both hospitals. Partial weight-bearing in this study was considered to be weight-bearing with a walker or bilateral side crutch and assigned to patients that could support less than half of their body weight in the affected leg. Partial weight-bearing was encouraged three months after surgery. Full weight-bearing in this study was encouraged six months after surgery.

Postoperatively, patients were seen in the outpatient clinic. Postoperative Harris hip scores were recorded six months after surgery by orthopedic rehabilitative clinicians in the outpatient clinic. Follow-up was performed every three months. The primary endpoint of follow-up in this study was the development of postoperative ONFH, preserving femoral head surgery due to preliminary ONFH and arthroplasty.

\section{Statistical analysis}

Continuous variables were described using median and standard error (SE). Categorical variables were presented using frequency and percentages. Statistical comparisons between groups were carried out using the MannWhitney $\mathrm{U}$ test for continuous variables and the Chisquare test for categorical variables. Cox regression models were used to determine hazard ratios (HRs) and identify independent prognostic factors. Logistic regression analyses were performed to identify risk factors associated with postoperative ONFH. The nomogram for prediction of postoperative ONFH was constructed using the independent predictors identified in the multivariable logistic analysis, which was derived from the primary training cohort $(n=330)$. The discrimination ability of the nomogram was assessed by receiver operating characteristic (ROC) curve analysis. The calibration curve was evaluated using a calibration plot. The clinical usefulness of the nomogram was measured by decision curve analysis (DCA). Performance of this nomogram was assessed in the validation cohort $(n=140)$.

A two-tailed $\mathrm{P}$ value under 0.05 was considered significant in this study. Variables with an original significance level of $\mathrm{P}<0.10$ in univariate analysis were entered into multivariable analyses. Model selection was based on the forward and backward stepwise method using the likelihood ratio test with Akaike information criterion as the stopping rule. The final model selected was the one with the smallest Akaike information criterion. All analyses were performed using $\mathrm{R}$ software (version 3.5.2).

\section{Results}

\section{Patient baseline}

Altogether, there were 470 patients who met the inclusion criteria for this study. Clinical baseline characteristics of the patients are listed in Table 1. There were 252 (53.6\%) male and 218 (46.4\%) female patients, with a median age of $45.8 \pm 13.6$ years at the time of diagnosis. Eventually, 141 patients were diagnosed with postoperative $\mathrm{ONFH}$ at the last follow-up. The incidence of ONFH in this cohort was $30 \%$. The BMI in the ONFH and non-ONFH cohorts was $23.2 \pm 3.02$ and $22.5 \pm 2.95$, respectively $(\mathrm{P}=0.010)$. 
Table 1 Patient characteristics

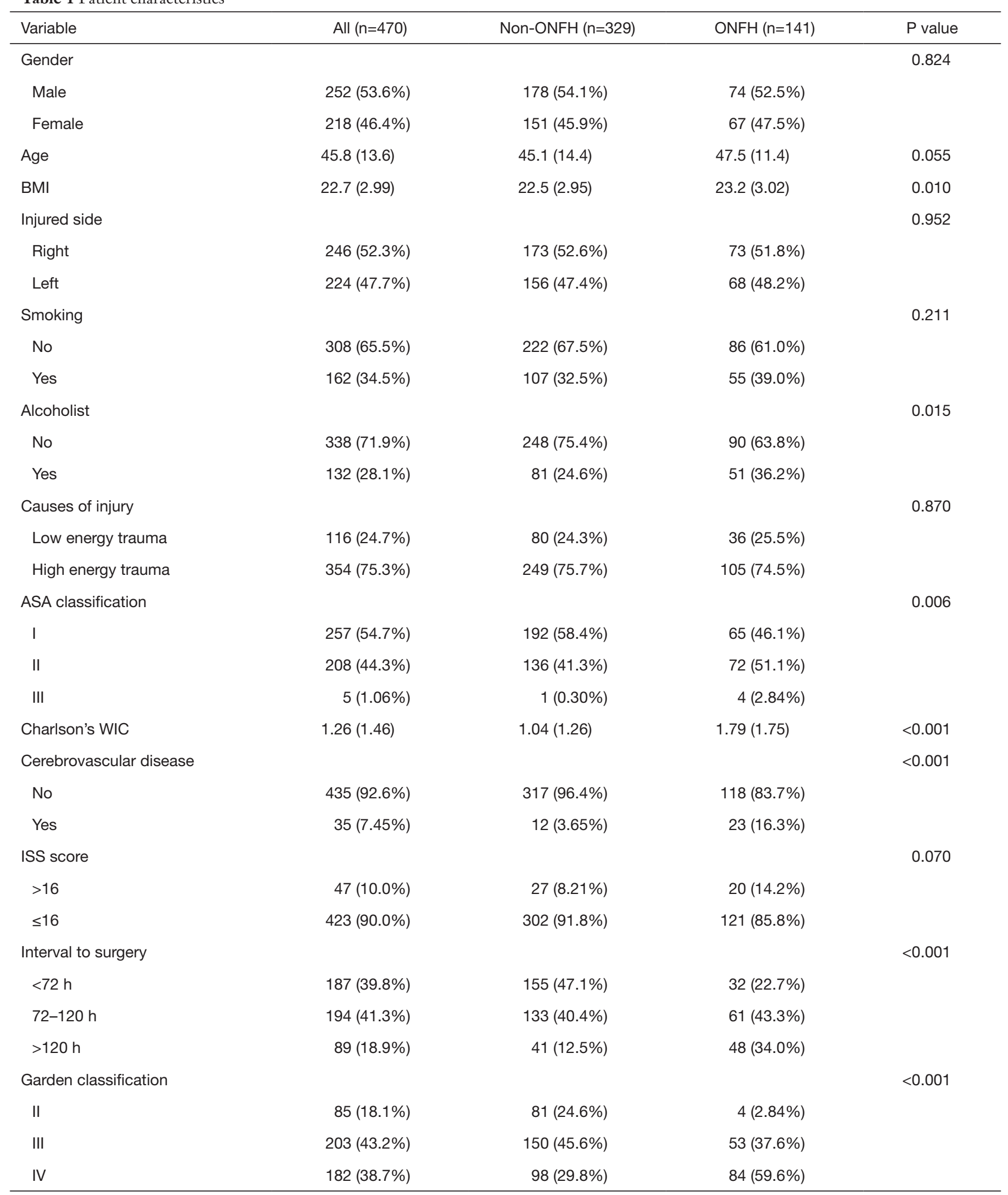

Table 1 (continued) 
Table 1 (continued)

\begin{tabular}{|c|c|c|c|c|}
\hline Variable & All $(n=470)$ & Non-ONFH $(n=329)$ & ONFH $(n=141)$ & $P$ value \\
\hline Garden Index & & & & $<0.001$ \\
\hline I & $84(17.9 \%)$ & $71(21.6 \%)$ & $13(9.22 \%)$ & \\
\hline II & $148(31.5 \%)$ & $124(37.7 \%)$ & $24(17.0 \%)$ & \\
\hline IV & $100(21.3 \%)$ & $41(12.5 \%)$ & $59(41.8 \%)$ & \\
\hline WBC & $7.21(2.33)$ & $7.27(2.30)$ & $7.07(2.38)$ & 0.396 \\
\hline RBC & $4.33(0.58)$ & $4.33(0.59)$ & $4.33(0.57)$ & 0.911 \\
\hline PLT & $184(60.3)$ & $178(56.6)$ & $197(66.7)$ & 0.004 \\
\hline Interval to part-weight bearing & & & & $<0.001$ \\
\hline$<1 \mathrm{~m}$ & 27 (5.74\%) & $5(1.52 \%)$ & $22(15.6 \%)$ & \\
\hline $1-3 m$ & $167(35.5 \%)$ & $116(35.3 \%)$ & $51(36.2 \%)$ & \\
\hline $3-6 \mathrm{~m}$ & $261(55.5 \%)$ & $195(59.3 \%)$ & $66(46.8 \%)$ & \\
\hline$>6 \mathrm{~m}$ & $15(3.19 \%)$ & $13(3.95 \%)$ & $2(1.42 \%)$ & \\
\hline Interval to full-weight bearing & & & & 0.480 \\
\hline$<3 \mathrm{~m}$ & $36(7.66 \%)$ & $22(6.69 \%)$ & $14(9.93 \%)$ & \\
\hline
\end{tabular}

ONFH, osteonecrosis of femoral head; BMI, body mass index; ASA, the American Society of Anesthesiologists; WIC, Charlson's weighted index of comorbidities; ISS, injury severity score; WBC, white blood cell; RBC, red blood cell; PLT, platelet; Hb, hemoglobin; ALB, albumin.

Alcohol use was higher in the group of patients that developed ONFH than in that without ONFH (36.2\% vs. $24.6 \% ; \mathrm{P}=0.015)$. Presence of cerebrovascular disease of patients with $\mathrm{ONFH}(16.3 \%)$ was significantly higher in patients with ONFH than those without ONFH $(16.3 \%$ vs. $3.65 \%$; $\mathrm{P}<0.01)$. Significant differences were also found in ASA classification, WIC, interval to surgery, Garden classification, Pauwels angle, Garden index, blood platelet count (PLT), interval to partial weight-bearing, and six-month postoperative Harris hip score between patients with $\mathrm{ONFH}$ and those without $\mathrm{ONFH}$ (all $\mathrm{P}<0.05$; Table 1). There was no statistical difference in age, sex, side of injury, tobacco use, cause of injury, and interval to full weight- bearing between the two groups.

\section{Development of an individualized nomogram}

In univariate logistic regression analyses, age, BMI, alcohol use, cerebrovascular disease, WIC, ASA classification, interval to surgery, Garden classification, Pauwels angle, Garden Index, interval to part-weight bearing, and six-month postoperative Harris hip score were found to be significant factors associated with $\mathrm{ONFH}$ in the training cohort (Table 2). In the final multivariable logistic regression model, cerebrovascular disease, interval to surgery, Garden classification, Garden index, interval to partial weight- 
Table 2 Univariate and stepwise multivariate logistic analysis of risk factors for ONFH

\begin{tabular}{|c|c|c|c|c|}
\hline Variable & \multicolumn{2}{|c|}{ Univariate analysis } & \multicolumn{2}{|c|}{ Multivariate analysis } \\
\hline Age (continuous) & $1.024(1.006-1.044)$ & 0.010 & Not selected & - \\
\hline Gender, female & $1.159(0.713-1.881)$ & 0.550 & - & - \\
\hline BMI (continuous) & $1.112(1.025-1.209)$ & 0.011 & Not selected & \\
\hline Alcoholist, yes & $1.743(1.042-2.901)$ & 0.033 & $2.342(0.796-7.138)$ & 0.124 \\
\hline Cerebrovascular disease, yes & $5.357(2.318-13.13)$ & $<0.001$ & $7.356(1.143-74.43)$ & 0.053 \\
\hline WIC (continuous) & $1.504(1.283-1.778)$ & $<0.001$ & Not selected & \\
\hline ISS score, $\leq 16$ & $0.578(0.271-1.276)$ & 0.162 & - & - \\
\hline ASA classification & & & Not selected & - \\
\hline 1 & Reference & & - & - \\
\hline II & $2.226(1.371-3.742)$ & 0.001 & - & - \\
\hline III & $16.35(2.33-325.5)$ & 0.014 & - & - \\
\hline \multicolumn{5}{|l|}{ Interval to surgery } \\
\hline$<72 \mathrm{~h}$ & Reference & & Reference & \\
\hline $72-120 \mathrm{~h}$ & $2.226(1.240-4.078)$ & 0.008 & $1.811(0.612-5.659)$ & 0.291 \\
\hline Pauwells angle (continuous) & $1.030(1.013-1.047)$ & $<0.001$ & Not selected & - \\
\hline \multicolumn{5}{|l|}{ Garden index } \\
\hline I & Reference & & Reference & \\
\hline II & $0.664(0.271-1.665)$ & 0.372 & $5.297(0.906-49.14)$ & 0.092 \\
\hline III & $1.879(0.859-4.389)$ & 0.127 & $13.07(2.269-121.16)$ & 0.009 \\
\hline IV & $5.935(2.670-14.184)$ & $<0.001$ & 18.58 (2.944-185.48) & 0.005 \\
\hline \multicolumn{5}{|l|}{ Interval to part-weight bearing } \\
\hline$<1 \mathrm{~m}$ & Reference & & & \\
\hline $1-3 \mathrm{~m}$ & $0.110(0.029-0.327)$ & $<0.001$ & $0.221(0.010-2.034)$ & 0.054 \\
\hline $3-6 \mathrm{~m}$ & $0.077(0.020-0.224)$ & $<0.001$ & $0.076(0.004-0.574)$ & 0.030 \\
\hline$>6 \mathrm{~m}$ & $0.053(0.006-0.296)$ & 0.002 & $0.055(0.008-0.303)$ & $<0.001$ \\
\hline
\end{tabular}

Table 2 (continued) 
Table 2 (continued)

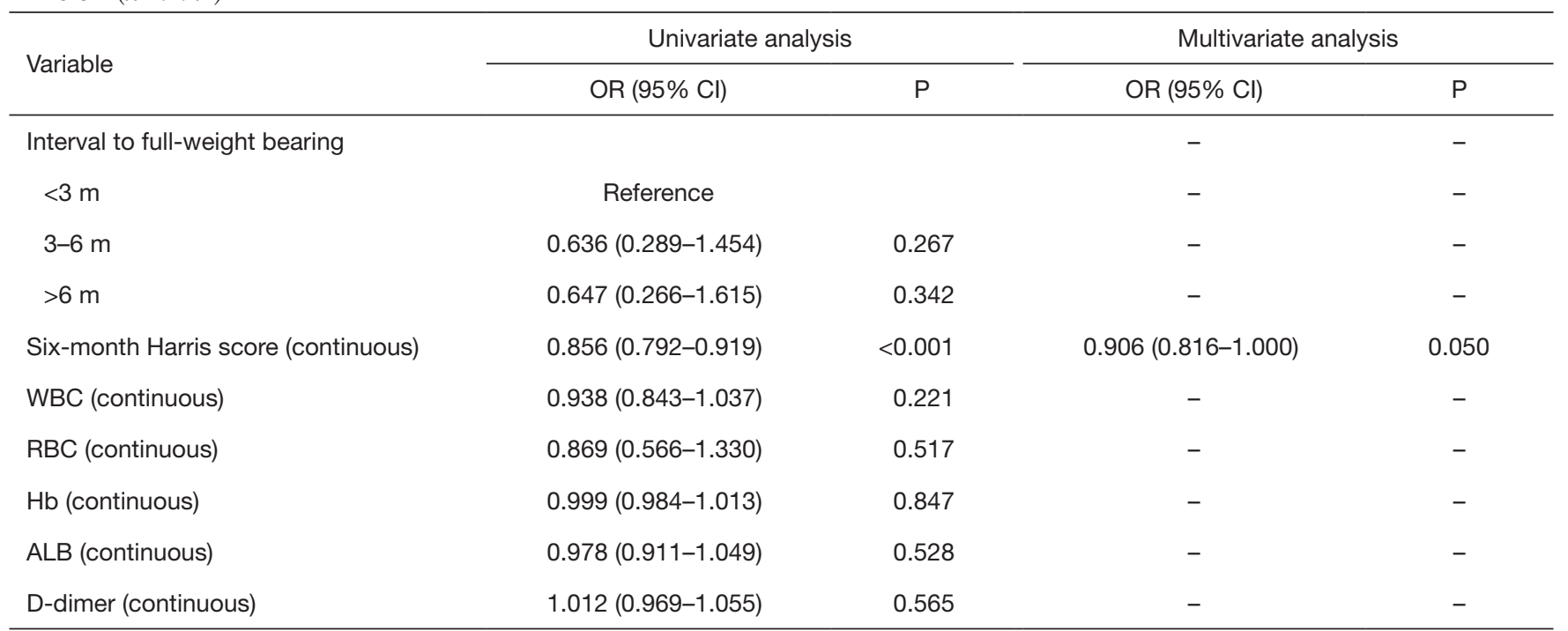

The variables with a $\mathrm{P}<0.1$ in univariate analyses were entered into multivariate step-wise analysis. ONFH, osteonecrosis of femoral head; BMI, body mass index; WIC, Charlson's weighted index of comorbidities; ISS, injury severity score; WBC, white blood cell; RBC, red blood cell; PLT, platelet; Hb, hemoglobin; ALB, albumin.

bearing, and six-month postoperative Harris hip score were identified as independent predictors of ONFH (Table 2). A nomogram for predicting postoperative ONFH was constructed with these independent predictors based on the multivariable model (Figure 1).

\section{Validation of the nomogram}

The calibration curve of the predictive nomogram for postoperative ONFH showed good agreement between prediction and observation in the primary training cohort (Figure 2A). We tested the nomogram independently in a validation cohort to validate its performance in predicting postoperative $\mathrm{ONFH}$ in patients with femoral fractures. Good calibration was observed for the probability of postoperative ONFH in the validation cohort (Figure $2 B$ ). The area under the curve (AUC) for the prediction nomogram was 0.865 (95\% CI, 0.821-0.905) for the primary training cohort (Figure $3 A$ ). The nomogram yielded an AUC of 0.877 (95\% CI, 0.817-0.937) for the validation cohort (Figure 3B).

\section{Clinical utility of the nomogram for predicting postoperative $\mathrm{ONFH}$}

To use the nomogram in the clinic, a vertical line is drawn to the top point row to assign points to each variable.
The total number of points is calculated afterwards, and a vertical line is drawn downward from the total point row to obtain the probability of postoperative ONFH. DCA for the predictive nomogram in the training and validation cohorts is presented in Figure 4. DCA indicates that, when the high-risk threshold for a doctor or patient is within a range of $0.2-0.9$ in the training cohort (Figure 4A), using the nomogram adds more net benefit than intervening either all or no patients. DCA of the nomogram in the validation cohort showed a similar clinical usefulness to that of the training cohort. After comparing the nomogram to the null model for its added value, we found it is applicable when the thresholds are in the range of $0.2-0.82$ in the validation cohort, due to the net benefit (Figure 4B).

\section{Discussion}

In this study, we developed and validated a predictive clinical nomogram for postoperative ONFH in patients with femoral neck fractures and cannulated screw fixation. Predictive factors were incorporated into the preliminary prediction and intervention of $\mathrm{ONFH}$ : cerebrovascular disease, interval to surgery, Garden classification, Garden Index, interval to partial weight-bearing, and six-month postoperative Harris hip score.

ONFH after internal fixation is a process caused by multiple factors, injury and fracture patterns having the 


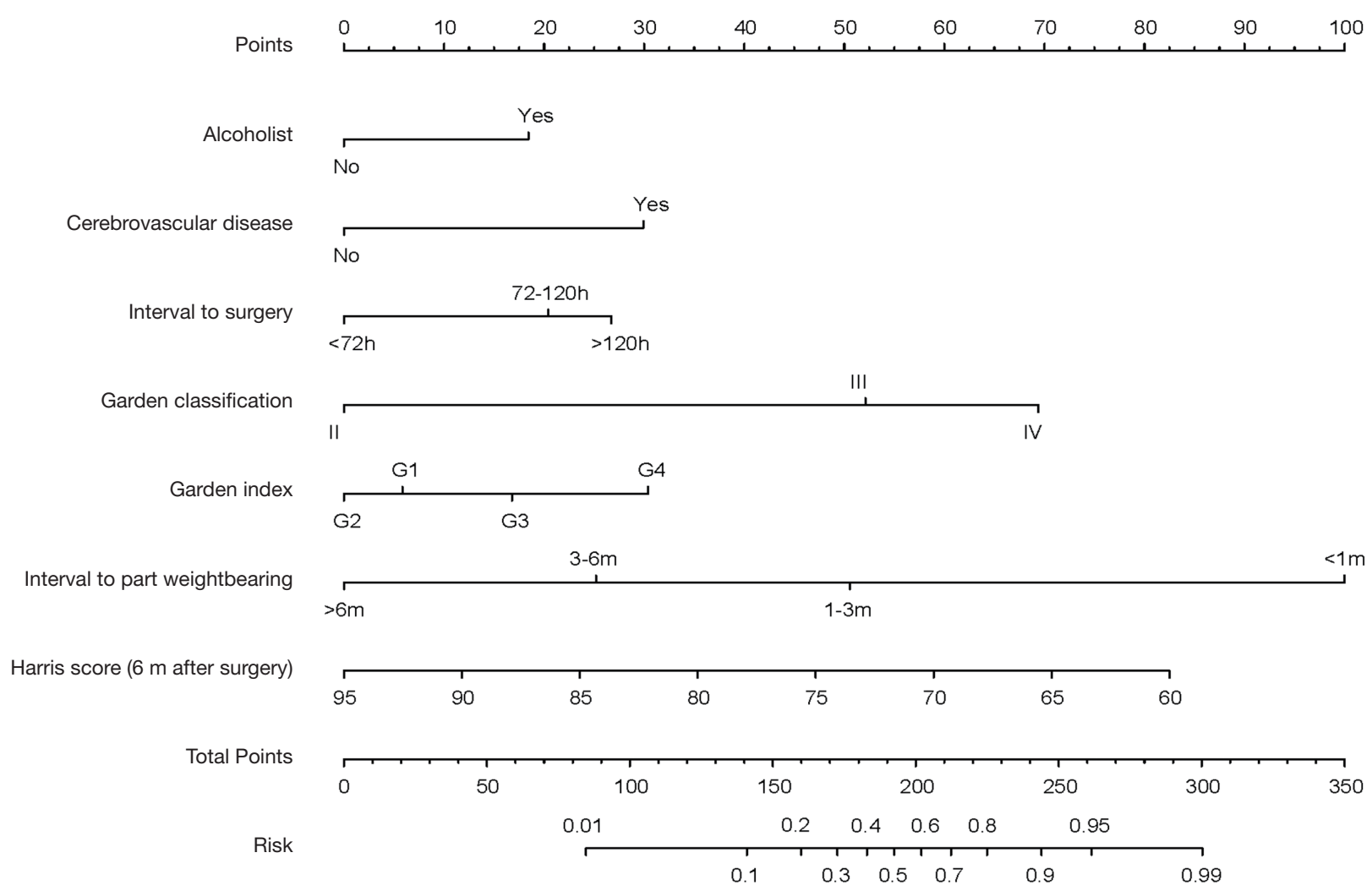

Figure 1 The nomogram for the postoperative ONFH with CS fixation. The nomogram was developed in the training cohort, with the alcoholism, cerebrovascular disease, interval to surgery, Garden classification, Garden index, interval to part weightbearing and Harris score (six-month after surgery) incorporated. ONFH, osteonecrosis of femoral head; CS, cannulated screw.

highest prognostic value. At least two mechanisms have been identified as major causes of avascular necrosis associated with injury and fracture patterns. High-energy fractures are characterized by complete displacement, which destroys the retinacular vessels in the trabeculae and the nourishing arteries of the femoral head, such as the medial and lateral circumflex femoral artery (24). This mechanism seems undisputed and is determined instantaneously by the injury. For more severe fractures-Garden III/ IV and high Pauwels angle fractures-there is a higher incidence of complications following femoral neck fracture fixation, including fracture nonunion and ONFH $(25,26)$. Early predictive studies on ONFH considered fracture classification to be the main factor, and it has been widely applied in clinical practice. For example, internal fixation is still considered an appropriate treatment for Garden I/ II or nondisplaced fractures in the elderly. Arthroplasty is highly recommended to avoid ONFH in Garden III/IV or high shear fractures (6). Another mechanism is the rapid increase in capsule pressure after injury, including bleeding at the fracture site and deformation caused by fracture displacement, leading to vascular pressure occlusion and perfusion of the femoral head (27). A super-selective arteriography study revealed that traction and internal rotation increase the intracapsular pressure of the hip joint, leading to ONFH (28). Thus, preoperative traction is not recommended in hospitals, including those in this study, so as to avoid the development of ONFH after femoral neck fracture, which was proved by DSA (29).

The injury-to-surgery interval was considered a main factor in predicting ONFH, but recent studies and systematic reviews showed that it did not significantly affect the development of ONFH (30). While the 12-hour period after fracture is generally considered to be the boundary between early and late fixation, the injury-to-surgery interval ranged from 3 to 18 days may not be associated with the incidence of complications in young patients (31-33). However, the preoperative interval was an independent predictor in this study. We found that a preoperative interval of more than 120 hours was of significant predictive value, 

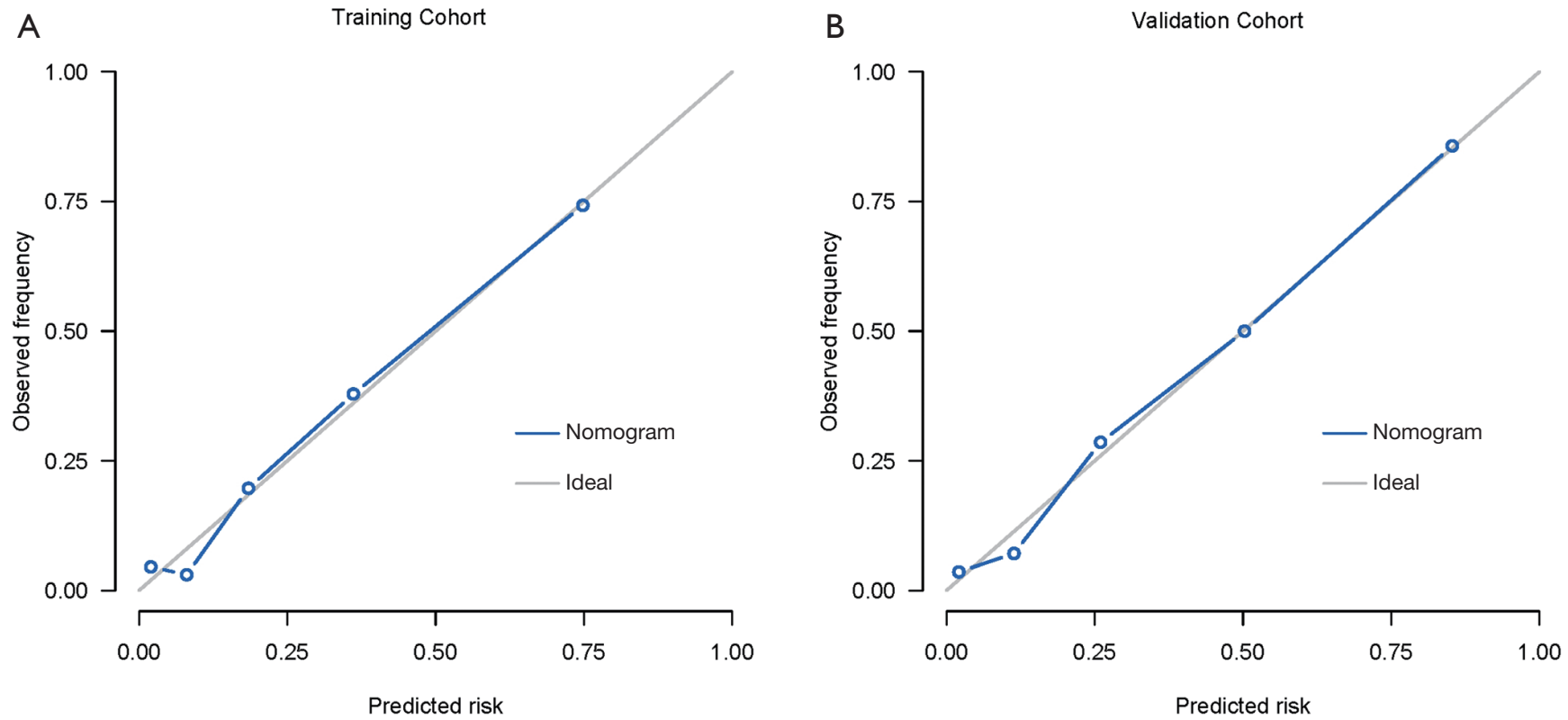

Figure 2 Calibration curves of the predictive nomogram in each cohort. (A) Calibration curve of the radiomics nomogram in the training cohort. (B) Calibration curve of the radiomics nomogram in the validation cohort. Calibration curves illustrate the calibration of the predictive nomogram in terms of agreement between the predicted risk of postoperative ONFH and detected outcomes. The diagonal grey line represents a perfect prediction by an ideal model. The dotted blue lines represent the predictive performance of the nomogram. The closer the dotted line fit is to the ideal line, the better the predictive accuracy of the nomogram is. ONFH, osteonecrosis of femoral head.
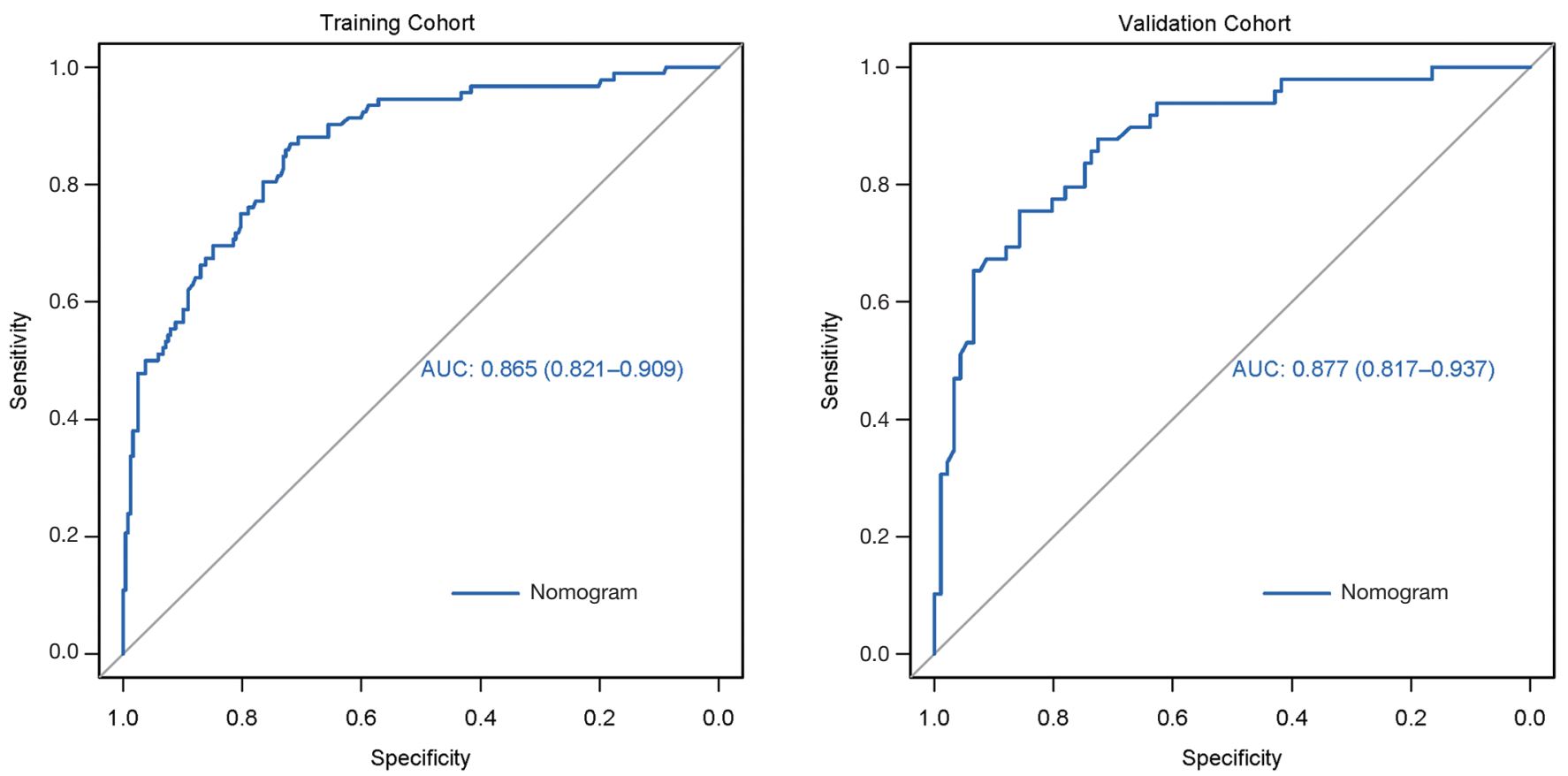

Figure 3 ROC curves of the nomogram in each cohort. (A) ROC curve of the nomogram in the training cohort; (B) ROC curve of the nomogram in the validation cohort. ROC, receiver operating characteristic. 

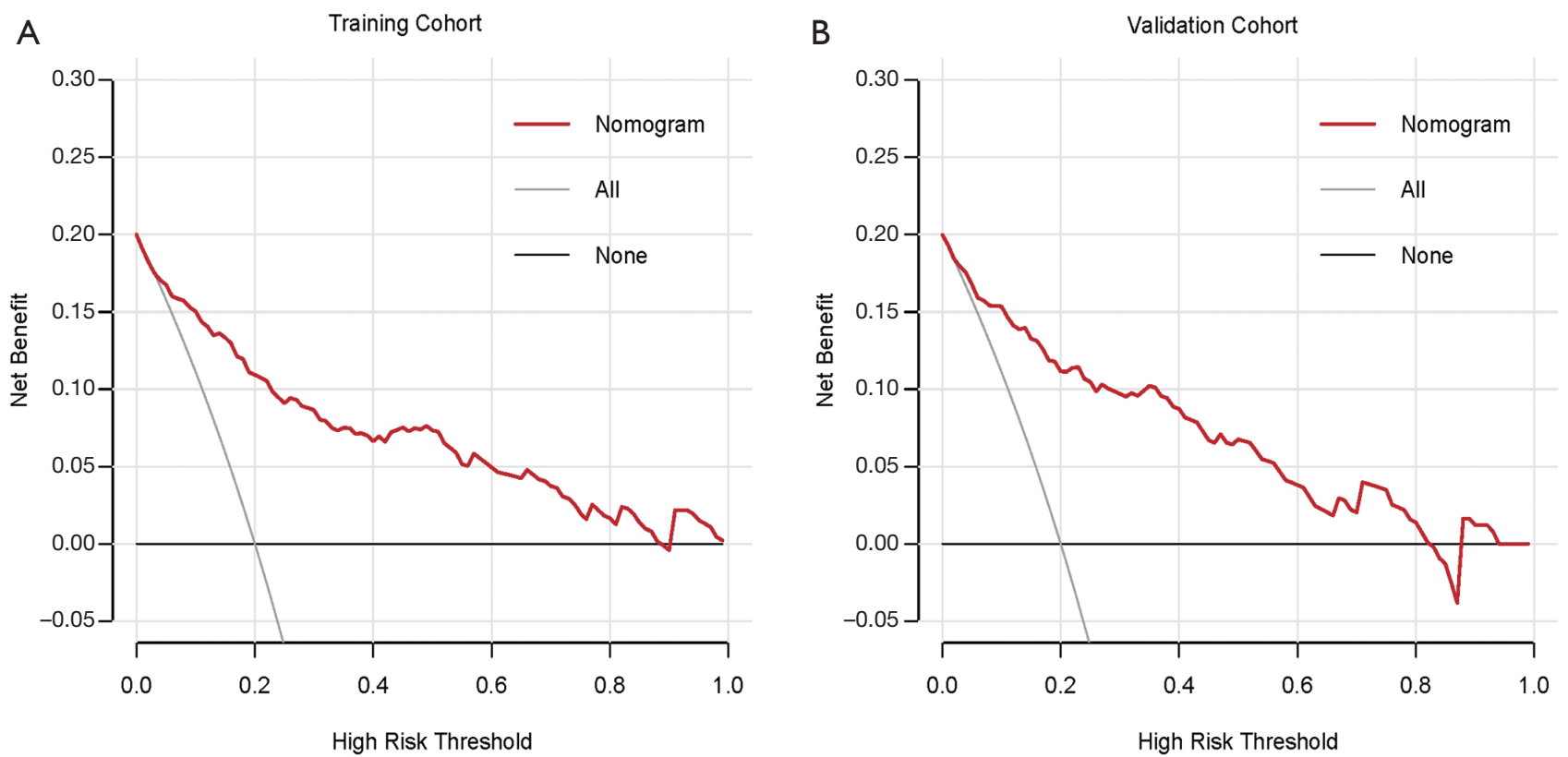

Figure 4 Decision curve analysis for the nomogram in each cohort. (A) DCA in the training cohort; (B) DCA in the validation cohort. The $y$-axis represents the net benefit. The red lines represent the predictive nomogram. The blue lines represent the hypothesis that all patients developed postoperative ONFH after CSs fixation. The black lines represent the hypothesis that no patients developed postoperative ONFH. The $\mathrm{x}$-axis represents the high-risk threshold probability. The net benefit was calculated by subtracting the proportion of all patients who are false positive from the proportion who are true positive, weighting by the relative harm of forgoing treatment compared with the negative consequences of an unnecessary treatment. DCA, decision curve analysis; ONFH, osteonecrosis of femoral head; CS, cannulated screw.

while an interval to reduction of less than 72 hours might avoid the occurrence of ONFH. This is in agreement with studies that suggest there is a decrease in the development of nonunion and ONFH by fixation within 12-24 hours after injury in patients under 60 years of age (34-36).

Satisfactory reduction quality and stable internal fixation are key to surgery that restores perfusion at the fracture site to promote fracture healing and avoid the development of ONFH (37). We used conventional anteroposterior and lateral position to observe the alignment of fracture reduction - the Garden Index-and determine the quality of fracture reduction (38). Different types of reduction classification systems are based on restoring the normal femoral neck anatomical structure (39). In recent years, a few studies have used positron emission tomography/CT (PET/CT) and other imaging indicators to observe blood perfusion after reduction, in order to evaluate the effect of reduction on the prediction of femoral head necrosis $(40,41)$. These studies predicted necrosis by detecting the blood supply to the femoral head before and after internal fixation for temporal variations in blood flow following femoral neck fracture. In a sense, anatomic reduction and fixation provide the basis for restoring blood supply. Thus, orthopedic surgeons need to consider choosing internal fixation to maintain a stable fixation relationship and avoid implant failure. In particular, cannulated screw fixation may not provide satisfactory biomechanical stability in shear fractures with a large Pauwels angle. Recent advances in buttress plate fixation and the femoral neck system may address this problem by avoiding necrosis of the femoral head due to loss of reduction (42-44). Our nomogram showed that achieving a satisfactory reduction index was one of the ways to reduce the risk of $\mathrm{ONFH}$ in more displaced fractures.

There is still no definite conclusion on the relationship between the time until weight-bearing, mobilization after internal fixation, and postoperative necrosis of the femoral head. Under the guidance of enhanced recovery after surgery, it seems that early functional exercise leads to more satisfactory functional outcomes (36). In low Pauwels angle fractures, early weight-bearing may convert compressive stress into forces that promote healing. 
In shearing fractures, premature weight-bearing may challenge the biological stability of internal fixation. At the same time, different hip joint positions during functional exercise change the intra-capsular pressure, which is potentially associated with ONFH $(27,45)$. Compared with arthroplasty, patients with internal fixation had prolonged weight-bearing time and poorer functional outcome (46). This difference may depend on postoperative fracture healing and range of motion. Some studies suggest that patients with internal fixation require at least 12 weeks before full weight-bearing, which will undoubtedly affect the function of the hip joint $(47,48)$. Good functional outcome but not regained health-related quality of life was also found in young fractures (49). Postoperative rehabilitation plans for patients with femoral neck fractures require surgeons and orthopedic rehabilitation physicians to make individualized recommendations for patients based on fracture patterns and internal fixation. In this study, we used partial weight-bearing time and full weight-bearing time as independent predictors. We found that partial weightbearing time, when performed with bilateral crutches, was a predictor of ONFH. This means that delayed weightbearing may reduce the predicted incidence of ONFH in patients with unstable fractures or in patients with a high predictive incidence of $\mathrm{ONFH}$ due to multiple preoperative factors.

The existing prediction model-including imaging, hemodynamics, and postoperative function outcomeshas been studied to predict the occurrence of ONFH from multiple dimensions to provide a basis for early intervention (24,50-53). We developed this nomogram for individualized prediction based on common and widely available clinical risk factors. For example, joint replacement can be directly selected to avoid the occurrence of ONFH in patients predicted by the model to be at high risk of necrosis, taking also into account the patient's wishes. Patients with high predictive value due to fracture pattern factors can improve the score of fracture reduction and reduce the probability of ONFH by special reduction techniques, open reduction, and more stable fixation methods such as the use of the buttress plate or femoral neck system. In addition to predicting the preoperative risk factors for ONFH, intervention can be carried out to improve postoperative variables such as weight-bearing, based on the preoperative score of the patient. Combined with the predictive incidence scale, $\mathrm{ONFH}$ risk prediction in the perioperative period may achieve a higher predictive value. Some studies also suggested that preoperative factors, such as blood flow examination and imaging variables, did not have predictive value, pointing to postoperative variables as additional important predictive risk factors $(40,41)$. Inclusion of some factors was controversial and not considered in our study. For example, in recent years, several studies have suggested retaining internal fixation as a way to reduce the incidence of refracture and necrosis (54). In clinical practice, we also recommend patients to avoid removal of internal fixation, which results in a minimal amount of removal surgeries. At the same time, some patients received removal surgery in local hospitals, which made it difficult for us to collect necessary surgical information. A few factors have no clear predictive value because of the pre-screening that takes place in hospitals. For example, age and gender were not significant predictors in our study or others. This may be because older patients tend to undergo arthroplasty, resulting in these studies including patients with a specific age range and gender, so these variables had no positive effect. However, factors that are not currently known to researchers cannot be included, which makes prediction methods always be one step behind. For example, although studies have shown that shortening of the femoral neck does not affect hip joint function, shortening may increase the occurrence of femoral head necrosis, even in Garden I fractures $(55,56)$. In recent years, artificial intelligence (AI) has been applied to identify elusive hip fractures (57). Unknown factors may be identified on the basis of big data through AI, which has the potential to improve the efficiency of prediction (58).

This study had some limitations. First, it was a retrospective cohort study, and selection bias cannot be ruled out. Due to the limited incidence of the disease in the population, the sample size and time of follow-up were slightly insufficient. Second, there was no external validation for the nomogram. Patient data from two independent hospitals were randomly assigned to establish and validate the nomogram, and our results showed the stability and applicability of the model; however, it would have been useful to use data from a third hospital as an external validation cohort. A prospective multicenter study is currently underway. Third, some controversial variables were not included in the study because they are either practically uncontrollable or have been proven to be invalid, such as removal of internal fixation, differences in surgery technique, and variability in brands and location of the internal fixation $(13,54)$. A recent study found that variability in location of constructs for the fixation following standard procedures did not significantly affect the outcome (59). 
Many patients also do not follow recommendations and undergo surgery in other hospitals, making the timing and surgical approach data not available.

\section{Conclusions}

In conclusion, we developed and validated a nomogram, based on perioperative variables, for individualized prediction of postoperative ONFH in patients with screw fixation. The risk of patients who are preoperatively predicted to be at high risk can be reduced by adjusting the intraoperative and postoperative variables, including time until weight-bearing. Postoperative high risk can be addressed by early intervention to improve function and quality of life, reducing the impact of ONFH. Models containing hybrid risk variables and using AI techniques for individual prediction need further study.

\section{Acknowledgments}

Funding: This work was supported by the National Natural Science Foundation of China (Grant No. 81871788), the project for Science and Technology leader of Anhui Province (Grant No. 2018H177), the Scientific Research Fund of Anhui Education (Grant No. 2017jyxm1097), the Anhui Provincial Postdoctoral Science Foundation (Grant No. 2019B302) and Key Research and Development Plan of Anhui Province (Grant No. 201904a07020097).

\section{Footnote}

Reporting Checklist: The authors have completed the TRIPOD reporting checklist. Available at http://dx.doi. org/10.21037/atm-20-4866

Data Sharing Statement: Available at http://dx.doi. org/10.21037/atm-20-4866

Peer Review File: Available at http://dx.doi.org/10.21037/ atm-20-4866

Conflicts of Interest: All authors have completed the ICMJE uniform disclosure form (available at http://dx.doi. org/10.21037/atm-20-4866). The authors have no conflicts of interest to declare.

Ethical Statement: The authors are accountable for all aspects of the work in ensuring that questions related to the accuracy or integrity of any part of the work are appropriately investigated and resolved. The study was conducted in accordance with the Declaration of Helsinki (as revised in 2013). This study was approved by the Ethics Committee of the First Affiliated Hospital of University of Science and Technology, the common ethics review institution of the First Affiliated Hospital of University of Science and Technology and the Southern Branch of the First Affiliated Hospital of the University of Science and Technology of China (No. 2020-P-049). The individual consent for this retrospective analysis was waived.

Open Access Statement: This is an Open Access article distributed in accordance with the Creative Commons Attribution-NonCommercial-NoDerivs 4.0 International License (CC BY-NC-ND 4.0), which permits the noncommercial replication and distribution of the article with the strict proviso that no changes or edits are made and the original work is properly cited (including links to both the formal publication through the relevant DOI and the license). See: https://creativecommons.org/licenses/by-nc-nd/4.0/.

\section{References}

1. Thorngren KG, Hommel AH, Norrman PO, et al. Epidemiology of femoral neck fractures. Injury 2002;33:C1-7.

2. Nauth A, Creek AT, Zellar A, et al. Fracture fixation in the operative management of hip fractures (FAITH): an international, multicentre, randomised controlled trial. Lancet 2017;389:1519-27.

3. Xu DF, Bi FG, Ma CY, et al. A systematic review of undisplaced femoral neck fracture treatments for patients over 65 years of age, with a focus on union rates and avascular necrosis. J Orthop Surg Res 2017;12:28.

4. Heetveld MJ RC, Frihagen F. Internal fixation versus arthroplasty for displaced femoral neck fractures: what is the evidence? J Orthop Trauma 2009;23:395-402.

5. Johansson T. Internal fixation compared with total hip replacement for displaced femoral neck fractures: a minimum fifteen-year follow-up study of a previously reported randomized trial. J Bone Joint Surg Am 2014;96:e46.

6. Wani IH, Sharma S, Latoo I, et al. Primary total hip arthroplasty versus internal fixation in displaced fracture of femoral neck in sexa- and septuagenarians. J Orthop Traumatol 2014;15:209-14.

7. Slobogean GP, Stockton DJ, Zeng B, et al. Femoral Neck 
Fractures in Adults Treated With Internal Fixation: A Prospective Multicenter Chinese Cohort. J Am Acad Orthop Surg 2017;25:297-303.

8. Wang T, Sun JY, Zha GC, et al. Analysis of risk factors for femoral head necrosis after internal fixation in femoral neck fractures. Orthopedics 2014;37:e1117-23.

9. Schweitzer D, Melero P, Zylberberg A, et al. Factors associated with avascular necrosis of the femoral head and nonunion in patients younger than 65 years with displaced femoral neck fractures treated with reduction and internal fixation. Eur J Orthop Surg Traumatol 2013;23:61-5.

10. Nikolopoulos KE, Papadakis SA, Kateros KT, et al. Long-term outcome of patients with avascular necrosis, after internal fixation of femoral neck fractures. Injury 2003;34:525-8.

11. Xie H, Wang B, Tian S, et al. Retrospective LongTerm Follow-Up Survival Analysis of the Management of Osteonecrosis of the Femoral Head With Pedicled Vascularized Iliac Bone Graft Transfer. J Arthroplasty 2019;34:1585-92.

12. Zhang CQ, Sun Y, Chen SB, et al. Free vascularised fibular graft for posttraumatic osteonecrosis of the femoral head in teenage patients. J Bone Joint Surg Br 2011;93:1314-9.

13. Ai ZS, Gao YS, Sun Y, et al. Logistic regression analysis of factors associated with avascular necrosis of the femoral head following femoral neck fractures in middle-aged and elderly patients. J Orthop Sci 2013;18:271-6.

14. Florschutz AV, Langford JR, Haidukewych GJ, et al. Femoral neck fractures: current management. J Orthop Trauma 2015;29:121-9.

15. Zheng J, Wang H, Gao Y, et al. A Study on the Evaluation of a Risk Score of Osteonecrosis of the Femoral Head Based on Survival Analysis. J Arthroplasty 202:S08835403(20)30824-X.

16. Reuling EM, Sierevelt IN, van den Bekerom MP, et al. Predictors of functional outcome following femoral neck fractures treated with an arthroplasty: limitations of the Harris hip score. Arch Orthop Trauma Surg 2012;132:249-56.

17. Slobogean GP, Sprague SA, Scott T, et al. Complications following young femoral neck fractures. Injury 2015;46:484-91.

18. Campenfeldt P, Al-Ani A, Hedstrom M, et al. Low BMD and high alcohol consumption predict a major re-operation in patients younger than 70 years of age with a displaced femoral neck fracture-A two -year follow up study in 120 patients. Injury 2018;49:2042-6.

19. Jiang J, Liu X, Lai B, et al. Correlational analysis between neutrophil granulocyte levels and osteonecrosis of the femoral head. BMC Musculoskelet Disord 2019;20:393.

20. Zhang C, Zhu X, Pei G, et al. Dyslipidaemia for patients with low-energy femoral neck fractures after the treatment of cancellous screws: a retrospective study with a 3-year minimum follow-up. BMC Musculoskelet Disord 2017;18:440.

21. Riaz O, Arshad R, Nisar S, et al. Serum albumin and fixation failure with cannulated hip screws in undisplaced intracapsular femoral neck fracture. Ann R Coll Surg Engl 2016;98:376-9.

22. Wang SH, Yang JJ, Shen HC, et al. Using a modified Pauwels method to predict the outcome of femoral neck fracture in relatively young patients. Injury 2015;46:1969-74.

23. Duffin M, Pilson HT. Technologies for Young Femoral Neck Fracture Fixation. J Orthop Trauma 2019;33 Suppl 1:S20-6.

24. Ehlinger M, Moser T, Adam P, et al. Early prediction of femoral head avascular necrosis following neck fracture. Orthop Traumatol Surg Res 2011;97:79-88.

25. Karaeminogullari O, Demirors H, Atabek M, et al. Avascular necrosis and nonunion after osteosynthesis of femoral neck fractures: effect of fracture displacement and time to surgery. Adv Ther 2004;21:335-42.

26. Schep NW, Heintjes RJ, Martens EP, et al. Retrospective analysis of factors influencing the operative result after percutaneous osteosynthesis of intracapsular femoral neck fractures. Injury 2004;35:1003-9.

27. Hosalkar HS, Varley ES, Glaser DA, et al. Intracapsular hip pressures in a porcine model: does position and volume matter? J Pediatr Orthop B 2011;20:278-83.

28. Wu K, Huang J, Wang Q. The Use of Superselective Arteriography in the Evaluation of the Influence of Intracapsular Hip Joint Pressure on the Blood Flow of the Femoral Head. Med Princ Pract 2016;25:123-9.

29. Xiao J, Yang XJ, Xiao XS. DSA Observation of Hemodynamic Response of Femoral Head With Femoral Neck Fracture During Traction: A Pilot Study. J Orthop Trauma 2012;26:407-13.

30. Gao YS, Ai ZS, Zhu ZH, et al. Injury-to-surgery interval does not affect postfracture osteonecrosis of the femoral head in young adults: a systematic review. Eur J Orthop Surg Traumatol 2013;23:203-9.

31. Jain R, Koo M, Kreder HJ, et al. Comparison of early and delayed fixation of subcapital hip fractures in patients sixty years of age or less. J Bone Joint Surg Am 2002;84:1605-12.

32. Araujo TPF, Guimaraes TM, Andrade-Silva FB, et 
al. Influence of time to surgery on the incidence of complications in femoral neck fracture treated with cannulated screws. Injury 2014;45:S36-9.

33. Razik F, Alexopoulos AS, El-Osta B, et al. Time to internal fixation of femoral neck fractures in patients under sixty years--does this matter in the development of osteonecrosis of femoral head? Int Orthop 2012;36:2127-32.

34. Papakostidis C, Panagiotopoulos A, Piccioli A, et al. Timing of internal fixation of femoral neck fractures. A systematic review and meta-analysis of the final outcome. Injury 2015;46:459-66.

35. Seyfettinoğlu F, Ersan O, Kovalak E, et al. Fixation of femoral neck fractures with three screws: results and complications. Acta Orthop Traumatol Turc 2011;45:6-13.

36. Kim JW, Byun SE, Chang JS. The clinical outcomes of early internal fixation for undisplaced femoral neck fractures and early full weight-bearing in elderly patients. Arch Orthop Trauma Surg 2014;134:941-6.

37. Wang Y, Ma JX, Yin T, et al. Correlation Between Reduction Quality of Femoral Neck Fracture and Femoral Head Necrosis Based on Biomechanics. Orthop Surg 2019;11:318-24.

38. Gregersen M, Krogshede A, Brink O, et al. Prediction of Reoperation of Femoral Neck Fractures Treated With Cannulated Screws in Elderly Patients. Geriatr Orthop Surg Rehabil 2015;6:322-7.

39. Karanicolas PJ, Bhandari M, Walter SD, et al. Interobserver reliability of classification systems to rate the quality of femoral neck fracture reduction. J Orthop Trauma 2009;23:408-12.

40. Kumar MN, Belehalli P, Ramachandra P. PET/CT study of temporal variations in blood flow to the femoral head following low-energy fracture of the femoral neck. Orthopedics 2014;37:e563-70.

41. Han S, Oh M, Yoon S, et al. Risk Stratification for Avascular Necrosis of the Femoral Head After Internal Fixation of Femoral Neck Fractures by Post-Operative Bone SPECT/ CT. Nucl Med Mol Imaging 2017;51:49-57.

42. Ye Y, Chen K, Tian K, et al. Medial buttress plate augmentation of cannulated screw fixation in vertically unstable femoral neck fractures: Surgical technique and preliminary results. Injury 2017;48:2189-93.

43. Putnam SM, Collinge CA, Gardner MJ, et al. Vascular Anatomy of the Medial Femoral Neck and Implications for Surface Plate Fixation. J Orthop Trauma 2019;33:111-5.

44. Stoffel K, Zderic I, Gras F, et al. Biomechanical Evaluation of the Femoral Neck System in Unstable Pauwels III Femoral Neck Fractures: A Comparison with the Dynamic
Hip Screw and Cannulated Screws. J Orthop Trauma 2017;31:131-7.

45. Yen CH, Leung HB, Tse PY. Effects of hip joint position and intra-capsular volume on hip joint intra-capsular pressure: a human cadaveric model. J Orthop Surg Res 2009; 4:8.

46. Ma HH, Chou TFA, Tsai SW, et al. Outcomes of internal fixation versus hemiarthroplasty for elderly patients with an undisplaced femoral neck fracture: a systematic review and meta-analysis. J Orthop Surg Res 2019;14:320.

47. Kubiak EN, Beebe MJ, North K, et al. Early weight bearing after lower extremity fractures in adults. J Am Acad Orthop Surg 2013;21:727-38.

48. Koval KJ, Sala DA, Kummer FJ, et al. Postoperative weight-bearing after a fracture of the femoral neck or an intertrochanteric fracture. J Bone Joint Surg Am 1998;80:352-6.

49. Campenfeldt P, Hedstrom M, Ekstrom W, et al. Good functional outcome but not regained health related quality of life in the majority of 20-69 years old patients with femoral neck fracture treated with internal fixation: A prospective 2-year follow-up study of 182 patients. Injury 2017;48:2744-53.

50. Liu Y, Li M, Zhang M, et al. Femoral neck fractures: prognosis based on a new classification after superselective angiography. J Orthop Sci 2013;18:443-50.

51. Bernaus M, Slobogean GP, Bzovsky S, et al. Early Radiographic Union Score for Hip Is Predictive of Femoral Neck Fracture Complications within 2 Years. J Orthop Trauma 2020;34:e195-202.

52. Palm H, Gosvig K, Krasheninnikoff $M$, et al. A new measurement for posterior tilt predicts reoperation in undisplaced femoral neck fractures: 113 consecutive patients treated by internal fixation and followed for 1 year. Acta Orthop 2009;80:303-7.

53. Frihagen F, Grotle M, Madsen JE, et al. Outcome after femoral neck fractures: a comparison of Harris Hip Score, Eq-5d and Barthel Index. Injury 2008;39:1147-56.

54. Xu JL, Liang ZR, Xiong BL, et al. Risk factors associated with osteonecrosis of femoral head after internal fixation of femoral neck fracture:a systematic review and metaanalysis. BMC Musculoskelet Disord 2019;20:632.

55. Nanty L, Canovas F, Rodriguez T, et al. Femoral neck shortening after internal fixation of Garden I fractures increases the risk of femoral head collapse. Orthop Traumatol Surg Res 2019;105:999-1004.

56. Haider T, Schnabel J, Hochpochler J, et al. Femoral shortening does not impair functional outcome after 
internal fixation of femoral neck fractures in non-geriatric patients. Arch Orthop Trauma Surg 2018;138:1511-7.

57. Badgeley MA, Zech JR, Oakden-Rayner L, et al. Deep learning predicts hip fracture using confounding patient and healthcare variables. NPJ Digit Med 2019;2:31.

58. Chee CG, Kim Y, Kang Y, et al. Performance of a Deep Learning Algorithm in Detecting Osteonecrosis of the

Cite this article as: Zhu W, Xie $\mathrm{K}$, Zhang $\mathrm{X}$, Yang J, $\mathrm{Xu}$ L, Zhu J, Fang S, Zhu C. Development and validation of a predictive nomogram for postoperative osteonecrosis of the femoral head with cannulated screws fixation. Ann Transl Med 2021;9(4):281. doi: 10.21037/atm-20-4866
Femoral Head on Digital Radiography: A Comparison With Assessments by Radiologists. AJR Am J Roentgenol 2019;213:155-62.

59. Li J, Wang M, Zhou J, et al. Finite element analysis of different screw constructs in the treatment of unstable femoral neck fractures. Injury 2020;51:995-1003. 